\title{
Uterine Corpus Undifferentiated Sarcoma
}

National Cancer Institute

\section{Source}

National Cancer Institute. Uterine Corpus Undifferentiated Sarcoma. NCI Thesaurus. Code C8972.

A rare, high grade sarcoma that arises from the endometrial stroma or myometrium without a specific type of differentiation. It was previously also known as high grade endometrial stromal sarcoma. In 2014, high grade endometrial stromal sarcoma was reclassified and is currently considered a distinct and rare neoplasm. Undifferentiated uterine sarcoma has a worse prognosis. 